\section{Capacitive approach to restore decoupling between channels for four-element MR coil array.}

\section{A. L. Perrier, D. Grenier, N. Ravel, P. Litaudon and O. Beuf}

Multi-channel coil arrays are increasingly being used to improve Signalto-Noise Ratio (SNR) in Magnetic Resonance Imaging (MRI). The decoupling between coils is an important parameter in array design. Indeed coupling between elements affects resonance frequency of each single coil and decreases it sensitivity. Many solutions were developed to achieve decoupling between elements of multi-channel coil array. In this paper, we present a capacitive solution to restore channel decoupling of a specific four-channel receiver coil array using common conductors. The principle of an effective decoupling was first demonstrated by circuit simulations of $|\mathrm{S}|$-parameters. A receive-only four-channel coil array was design for rat head MRI. Experimental $|S|-$ parameter measurements validated the proposed capacitive approach by restoring decoupling between elements and particularly between external loops.

Introduction: The Nuclear Magnetic Resonance (NMR) multichannel coil arrays introduced in 1990 [1] are used to increase sensitivity of MR coils while preserving a large volume of exploration. The basic idea is to use the juxtaposition or overlapping of several single loops. These loops are usually connected to low input impedance and low noise preamplifiers to reduce the coupling between the different elements of the array and to preserve SNR respectively. Recent works described other decoupling techniques such as strip transmission line array [2], overlap geometries [3] and capacitive decoupling networks [3, 4]. A different solution for two-channel coil arrays was also described. In this setup, single elements of the array are not juxtaposed or overlapped but elements are electrically jointed with a common conductor $[5,6,7]$. Based on this specific approach, a newly designed four-channel coil array was proposed [8].

Principle: The equivalent electrical circuit of a four-channel coil array based on common conductor architecture is presented in Fig.1 where inductors are representing conductor sections. At the operating frequency, $C_{d}$ and $C_{c}$ capacitance values are chosen to compensate imaginary impedance parts of the $L_{d}$ and $L_{c}$ inductance values, respectively. At the operating frequency, without $L_{\text {gnd }}$ parasitic inductances, these perfect compensations of impedance allow zeros of transmission between channels by deporting the ground plane to the black points labelled 3. This virtual ground plane between loops ensures the decoupling between channels [7, 8]. The three parasitic inductors $L_{\text {gnd }}$ coming from the experimental design of the coil array deteriorate zeros of transmission and lead to coupling between elements. In this paper, we propose a solution to restore the decoupling between elements by adding a $C_{\text {gnd }}$ capacitor in series with the central $L_{\text {gnd }}$ inductance. This solution is illustrated in "Simulated results" section with circuit simulations of Fig. 1 topology and in "Experimental results" section with measurement of a four-channel coil array described in "Design" section.

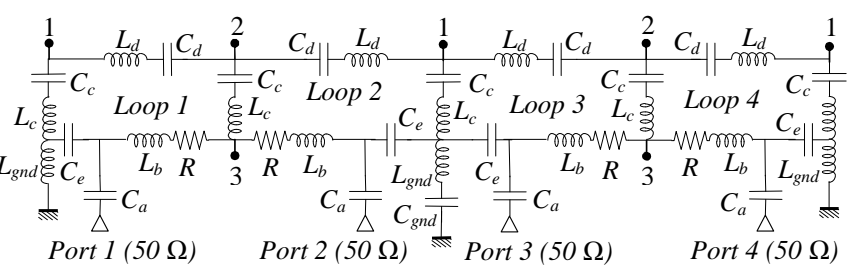

Fig. 1 Equivalent electrical circuit of a four channel coil array with common conductors.

Design: A specific four-channel receiver coil array was built on a flexible substrate and glued on a cylinder to fit the animal morphology. Loop inductors were realized with $35 \mu \mathrm{m}$ thick and $4 \mathrm{~mm}$ width section of copper tape. Each element of the coil consists in a rectangular loop with $20 \times 24 \mathrm{~mm}^{2}$ internal and $28 \times 32 \mathrm{~mm}^{2}$ external dimensions. To decouple the receiver array from the transmitter coil, each loop integrates an active decoupling circuit made with two DH80055 PIN diodes. Four $50 \Omega$ BNC cables were soldered at the four loops inputs to connect the coils with the acquisition data cabinet integrating transmit/receive switches and preamplifiers of MR system. The $C_{a}$ and $C_{e}$ were chosen to be adjustable elements for the four-channel coil array. Matching of each loop to $50 \Omega$ at $f_{0}=300 \mathrm{MHz}$ (proton Larmor frequency at $7 \mathrm{~T}$ static magnetic field) was realized using varicap diodes $\mathrm{BB} 149$. The capacitance values of these diodes ranged from $2 \mathrm{pF}$ to $22 \mathrm{pF}$ for a bias voltages between $-30 \mathrm{~V}$ and $0 \mathrm{~V}$. Fixed capacitors are non magnetic case A series 100 ATC capacitors. All fixe capacitors are experimentally adjusted to minimize all $\left|S_{i j}\right| \mathrm{dB}$ parameters. A photograph of the coil array is shown in Fig. 2.

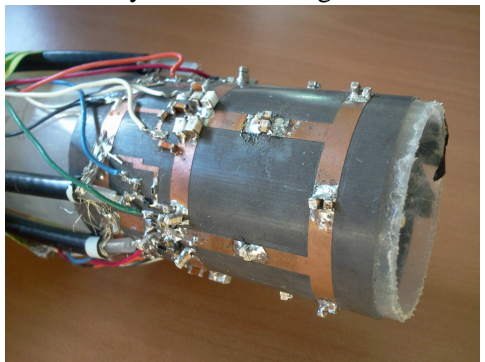

Fig. 2 Photograph of the four channel coil array built for rat head.
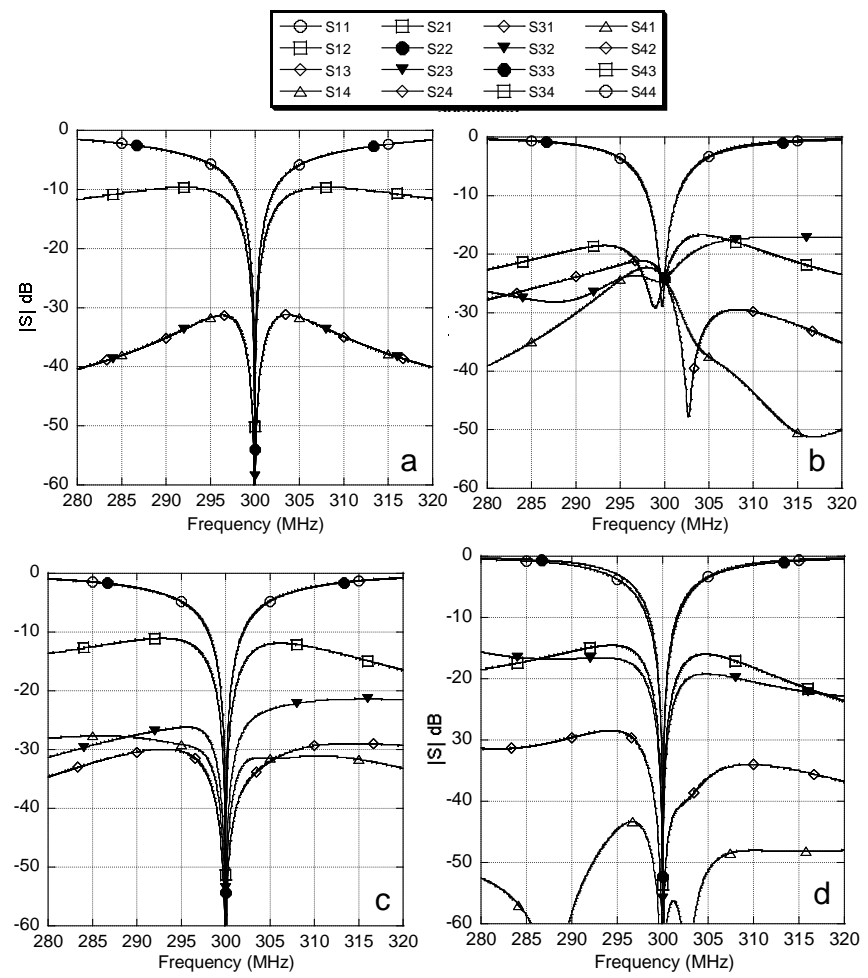

Fig. 3 Simulations of the four-channel coil array.

a Without parasitic inductance

$b$ With parasitic inductances $L_{g n d}$ fixed to $10 \mathrm{nH}$

c With capacitors $C_{\text {gnd }}$ in series with $L_{\text {gnd }}$ fixed to $28.1 \mathrm{pF}$

$d$ With one central capacitor $C_{g n d}$ in series with the central $L_{g n d}$ and fixed to $28.1 \mathrm{pF}$

Simulated results: Circuit simulations realised with Designer software. The influence of $L_{g n d}$ and $C_{g n d}$ component values on the $|S|$ parameters is shown in Fig. 3. Simulations were achieved for an arbitrarily chosen value of coil inductors and for a $300 \mathrm{MHz}$ operating frequency. The equivalent electrical circuit of Fig. 1 was simulated with all $L_{b}, L_{c}, L_{d}$ and $L_{g n d}$ values equal to $10 \mathrm{nH} ; R$ was fixed to $1.1 \Omega$. To compensate imaginary part of inductors at the operating frequency, capacitors $C_{c}$ and $C_{d}$ were fixed to $28.1 \mathrm{pF}$. $C_{a}$ and $C_{e}$ capacitors were fixed to $4.5 \mathrm{pF}$ and $24.2 \mathrm{pF}$ respectively. Fig. $3 a$ shows the ideal coil with perfect decoupling between channels at the operating frequency without $L_{g n d^{-}}$ $C_{\text {gnd }}$ components: all $\left|S_{i j}\right|$ parameters are lower than $-30 \mathrm{~dB}$ at the operating frequency. Fig. $3 b$ presents the decoupling deterioration due 
to the three $10 \mathrm{nH}$ parasitic inductances $L_{g n d}:\left|S_{i j}\right|_{\mathrm{dB}}$ parameters are increased at the operating frequency and curve minima are shifted. Fig. $3 c$ and Fig. $3 d$ present the restoration of decoupling between channels by additional $C_{\text {gnd }}$ capacitors equal to $28.1 \mathrm{pF}$. It can be noticed on simulated results that a single $C_{\text {gnd }}$ capacitor on central section (Fig. $3 c$ ) is sufficiency to restore an acceptable decoupling. This solution was chosen for practical realisation of the array.

Experimental results: $|S|$-parameter measurements of the four-channel coil array were carried out with an Agilent E5071C four-port VNA (Agilent Technologies Inc., Santa Clara, CA, USA). Results of coil array described in "Design" section are presented in Fig. $4 a$. $\left|S_{i i}\right|-$ parameters perform better than $-20 \mathrm{~dB}$ and characterizing each coil matching at the resonant frequency. $\left|S_{14}\right|$ parameter value is about $-7 \mathrm{~dB}$ which is not sufficient to decouple external loops. This $\left|S_{14}\right|$-value is accentuated by magnetic coupling compare to circuit simulation (Fig 3.b). All other coupling parameters are inferior to $-16 \mathrm{~dB}$ at the centre frequency. In Fig. $4 b$ a $24.8 \mathrm{pF}$ capacitor is added in series with the central $L_{g n d}$ of the coil array. At the operating frequency, the $\left|S_{i j}\right|-$ parameters are below $-20 \mathrm{~dB}$. These values allow the use of the coil array for MR imaging without coupling between channels.

\begin{tabular}{|c|c|c|c|}
\hline$\square$ & $\overrightarrow{-}$ & 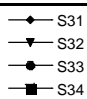 & 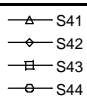 \\
\hline
\end{tabular}
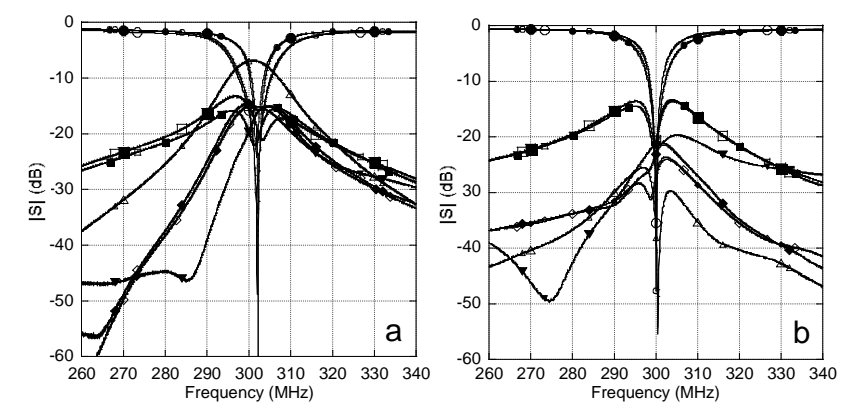

Fig. $4|S|$ parameters measurements of the four-channel coil array. a Without $C_{\text {gnd }}$

$b$ With one central capacitor $C_{\text {gnd }}=24.8 \mathrm{pF}$

Conclusion: A capacitive solution to achieve decoupling between elements of a particular four-channel coil array based on common conductors was first demonstrated by circuit simulations of an equivalent electrical circuit. The solution was validated by the experimental $|S|$-parameters measured on four-channel coil array dedicated to rat head: a coupling lower than $-20 \mathrm{~dB}$ was obtained between elements at the operating frequency.

Acknowledgments: The authors thank the Institut Féferatif des Neurosciences de Lyon (IFNL) for financial support. This work was supported by an ANR grant (\#ANR-07-NEURO-030).

A. L. Perrier, D. Grenier and O. Beuf (Université de Lyon, CREATIS, CNRS UMR 5220, Inserm U1044, INSA-Lyon, Université Lyonl, Villeurbanne, France)

E-mail: anne-laure.perrier@univ-lyon1.fr

N. Ravel and P. Litaudon (Centre de Recherche en Neurosciences de Lyon, CNRS UMR 5292, INSERM U1028, Université Lyon 1, France)

\section{References}

1 Roemer P. B., Edelstein W. A., Hayes C. E., Souza S. P. and Mueller O. M.: 'The NMR phased array', Magn Reson Med, 1990, 16, pp. 192225.

2 Adriany G., Van de Moortele P. F., Ritter J., Moeller S., Auerbach E. J., Akgun C., Snyder C. J., Vaughan T. and Ugurbil K.: 'A geometrically adjustable 16-channel transmit/receive transmission line array for improved RF efficiency and parallel imaging performance at 7 tesla', Magn Reson Med, 2008, 59, pp. 590-597.

3 Pinkerton R. G., Barberi E. A. and Menon R. S.: 'Noise properties of a NMR transceiver coil array', Journal of Magnetic Resonance, 2004, 171, pp. 151-156.

4 Zhang X. and Webb A.: 'Design of a capacitively decoupled transmit/receive NMR phased array for high field microscopy at 14.1 T', Journal of Magnetic Resonance, 2004, 170, pp. 149-155.

5 Gareis D., Neuberger T., Behr V. C., Jakob P. M., Faber C., Griswold M. A.: 'Transmit-receive coil-arrays at 17.6T, configurations for $\mathrm{H}-1$, Na-23, and P-31 MRI', Concepts Magn Reson Part B, Magn Reson Eng, 2006, 29B, pp. 20-27.

6 Dieringer M. A., Renz W., Lindel T., Seifert F., Frauenrath T., von Knobelsdorff-Brenkenhoff F., Waiczies H., Hoffmann W., Rieger J., Pfeiffer H., Ittermann B., Schulz-Menger J., and Niendorf T.: 'Design and Application of a Four-Channel Transmit/Receive Surface Coil for Functional Cardiac Imaging at 7T', Journal of Magnetic Resonance Imaging , 2011, 33, pp. 736-741.

7 Perrier A. L. , Grenier D., Pouzin A., Esclassan F., Ravel N., Litaudon P., Beuf O.: 'Design of a two-channel NMR coil using an impedance transformation approach', IEEE Sensors Journal, 2012, 12, pp. 18011808.

8 Perrier A.L., Goebel J.C., Pinzano A., Roeder E., Gillet P., Grenier D., and Beuf O., 'Design of a four-channel array coil for dual highresolution rat knee MR Imaging', International Society for Magnetic Resonance in Medicine, 2013. 